\title{
Radiation Field of a Square, Helical Beam Antenna
}

\author{
Knudsen, Hans Lottrup
}

Published in:

Journal of Applied Physics

Link to article, DOI:

10.1063/1.1702231

Publication date:

1952

Document Version

Publisher's PDF, also known as Version of record

Link back to DTU Orbit

Citation (APA):

Knudsen, H. L. (1952). Radiation Field of a Square, Helical Beam Antenna. Journal of Applied Physics, 23(4), 483-491. https://doi.org/10.1063/1.1702231

\section{General rights}

Copyright and moral rights for the publications made accessible in the public portal are retained by the authors and/or other copyright owners and it is a condition of accessing publications that users recognise and abide by the legal requirements associated with these rights.

- Users may download and print one copy of any publication from the public portal for the purpose of private study or research.

- You may not further distribute the material or use it for any profit-making activity or commercial gain

- You may freely distribute the URL identifying the publication in the public portal

If you believe that this document breaches copyright please contact us providing details, and we will remove access to the work immediately and investigate your claim 


\title{
Radiation Field of a Square, Helical Beam Antenna
}

\author{
H. LOTTRUP KNUDSEN \\ Microwave Laboratory, Royal Technical University of Denmark, Copenhagen, Denmark
}

(Received September 27, 1951)

\begin{abstract}
Rigorous formulas have been derived for the field from a square, helical antenna with a uniformly progressing current wave of constant amplitude. These formulas that have the advantage of great simplicity are of direct use for helical antennas in the meter band, where for practical reasons only square helices are used. Further, in connection with corresponding rigorous formulas for the field from a circular, helical antenna with a uniformly progressing current wave of constant amplitude the present formulas may be used for an investigation of the magnitude of the error introduced in Kraus' approximate calculation of the field from a circular, helical antenna by replacing this antenna with an "equivalent" square helix. This investigation is carried out by means of a numerical example. The investigation shows that Kraus' approximate method of calculation yields results in fair agreement with the results obtained from the rigorous formulas. A statement to the contrary made recently in the literature is shown to rest on a trivial error.
\end{abstract}

\section{INTRODUCTION}

$I^{\mathrm{N}}$ N $1947 \mathrm{Kraus}^{1}$ described a helical antenna with such dimensions that the field radiated from this antenna has a sharp maximum in the direction of the axis of the helix and is circularly polarized in this direction. As yet no complete theory of the field radiated from this antenna has been given. However, by measurements $\mathrm{Kraus}^{2,3}$ has found that the current distribution on a helical antenna with several turns with good approximation may be described as a uniformly progressing current wave with a constant amplitude. The problem of finding the field radiated by a helical antenna with this current distribution is mathematically well defined. Kraus $^{2,3}$ has given an approximate solution of this problem for a circular, helical antenna by first replacing the circular helix with an equivalent square helix and then making further mathematical approximations. The problem of the circular helix was solved rigorously by the author ${ }^{4}$ and was later solved independently by Kornhauser. ${ }^{5}$ In applying the rigorous formulas to a numerical example Kornhauser obtains a result which according to his statement differs considerably from the corresponding result obtained by using Kraus' approximate solution. However, it will be shown in this paper that there is a fair agreement between the results obtained by using Kraus' approximate formulas and by using the rigorous formulas. Kornhauser's erroneous statement rests on a trivial error that is pointed out in the following.

In the present paper rigorous expressions for the field from a square, helical antenna with a uniformly progressing current wave with constant amplitude will be derived. This work is essentially based on the last half of the author's aforementioned paper on circular and

\footnotetext{
${ }^{1}$ J. D. Kraus, Electronics 20, 109-111 (1947).

2 J. D. Kraus and J. C. Williamson, J. Appl. Phys. 19, 87-96 (1948).

3 J. D. Kraus, Proc. Inst. Radio Engrs. 37, 263-272 (1949).

${ }^{4} \mathrm{H}$. Lottrup Knudsen, The Field Radiated by Circular and Square, Belical Beam Antennas (Trans. Danish Acad. Tech. Sci., $1950)$, No. 8, 55 pp.

6 E. T. Kornhauser, J. Appl. Phys. 22, 887-891 (1951).
}

square helical antennas. ${ }^{4}$ The obtained result will be of direct use for helical antennas in the meter band, helical antennas for this wavelength region being constructed as square helices. Further, in connection with the rigorous formulas for a circular, helical antenna, a knowledge of the rigorous formulas for a square, helical antenna will make possible an investigation of the magnitude of the error introduced in the calculation of the field from a circular helix by replacing this with an "equivalent" square helix as is done by Kraus ${ }^{2,3}$ in his approximate method of calculation. This investigation will be carried out by applying the rigorous formulas for square and for circular helical antennas to a numerical example.

\section{FORMULATION OF THE PROBLEM}

Whereas a circular helix may be produced by folding a plane with a straight line on it into a circular cylinder, a "square" helix may be produced by folding a plane with a straight line on it onto a cylinder with a square cross section. In the present paper we set us the problem to calculate the field from a square helical antenna with a uniformly progressing current wave. For the sake of simplicity we confine ourselves to considering helical antennas with an integral number of turns. As shown by Kraus $^{2,3}$ a helical antenna with a progressing current wave may be considered an array of single turns. The array characteristic may be calculated by using wellknown formulas; the problem therefore reduces to that of investigating the field from a single turm of the helical antenna.

A single turn of a square helix is shown in Fig. 1. A rectangular coordinate system $(x, y, z)$ is introduced as shown in Fig. 1 and more clearly in Fig. 2, where the antenna is shown in two perpendicular projections. The antenna is made up of four pieces of straight wire connecting the points $(-b, 0,-2 l),(0,-b,-l),(b, 0,0)$, $(0, b, l)$, and $(-b, 0,2 l)$. The length of each of the straight pieces of line of which the antenna is composed is called $c$, the total length of a single turn $L$, and the 


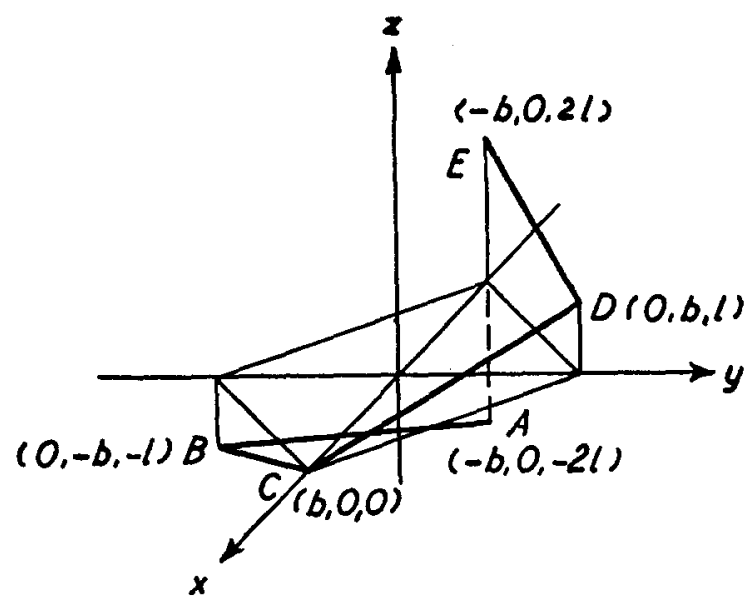

FIg. 1. Square, helical antenna with one turn.

pitch $S$. With these symbols we have

$$
\begin{aligned}
l / S & =c / L=1 / 4, \\
c & =\left(l^{2}+2 b^{2}\right)^{\xi} .
\end{aligned}
$$

Denoting the arc length of the antenna measured from the midpoint, $C$, of the wire and positive for $z>0$, by $s$, the current distribution on the antenna may be expressed by

$$
I=I_{0} e^{i \beta s} ;
$$

the parameter $\beta$ denotes the transmission coefficient for the current wave. Introducing a terminology used by Kraus $^{2,3}$ we set $\beta=k / p$, where $k$ is the intrinsic transmission coefficient of the medium surrounding the antenna.

\section{CALCULATION OF THE RADIATION FIELD}

We consider a current distribution in space with the density $J(\bar{r})$. Supposing that the current density differs from zero only in a finite domain we introduce a rectangular coordinate system $(x, y, z)$ with its origo in or near this domain and a spherical coordinate system $(r, \theta, \varphi)$ oriented in the usual way with respect to the first-mentioned system. Letting $\zeta=\eta^{-1}$ denote the intrinsic impedance of space, $r$ the distance from origo 0 to a point $P$ in the far zone, $\bar{\rho}$ a unit vector pointing in the direction from 0 to $P$, and $\theta$ and $\varphi$ the pole distance and the azimuth of $P$, we may express the electric field strength $\bar{E}$ and the magnetic field strength $\bar{H}$ at the point $P$ in the following way: $:^{4,6}$

$$
\begin{aligned}
& \bar{E}(r, \theta, \varphi)=T(r) \bar{F}(\theta, \varphi), \\
& \bar{H}(r, \theta, \varphi)=\eta \bar{\rho} \times \bar{E}(r, \theta, \varphi),
\end{aligned}
$$

with

$$
T=K i \omega e^{i k r} / r .
$$

In these equations $K$ denotes an arbitrary constant of which we shall despose later, whereas the spherical

* Time factor: $e^{-i \omega t}$.

-S. A. Schelkunoff, Electromagnetic Waves (D. van Nostrand Company, Inc., New York, 1943), pp. 331-336. coordinates of the vector $\bar{F}$ are expressed by

$$
\begin{aligned}
& F_{r}=0 \\
& F_{\theta}=F_{x} \cos \theta \cos \varphi+F_{y} \cos \theta \sin \varphi-F_{z} \sin \theta \\
& F_{\varphi}=-F_{x} \sin \varphi+F_{y} \cos \varphi .
\end{aligned}
$$

The rectangular coordinates of $\bar{F}$ are defined by

$$
\begin{array}{r}
F_{x}(\theta, \varphi)=\frac{1}{K} \frac{\mu}{4 \pi} \int J_{x}\left(\tilde{r}^{\prime}\right) \exp \left(-i k \bar{r}^{\prime} \cdot \bar{\rho}\right) d v^{\prime} \\
=\frac{1}{K} \frac{\mu}{4 \pi} \int J_{x}(x, y, z) \exp [-i k(x \sin \theta \cos \varphi \\
\quad+y \sin \theta \sin \varphi+z \cos \theta)] d x d y d z,
\end{array}
$$

and similar equations for $F_{y}$ and $F_{z}$. In the first one of the above integrals $\tilde{r}^{\prime}$ denotes the position vector of the point at which the current element $J_{x}\left(\tilde{r}^{\prime}\right) d v^{\prime}$ is situated. In the last integral the suffix has been dropped as no misunderstanding seems to be possible.
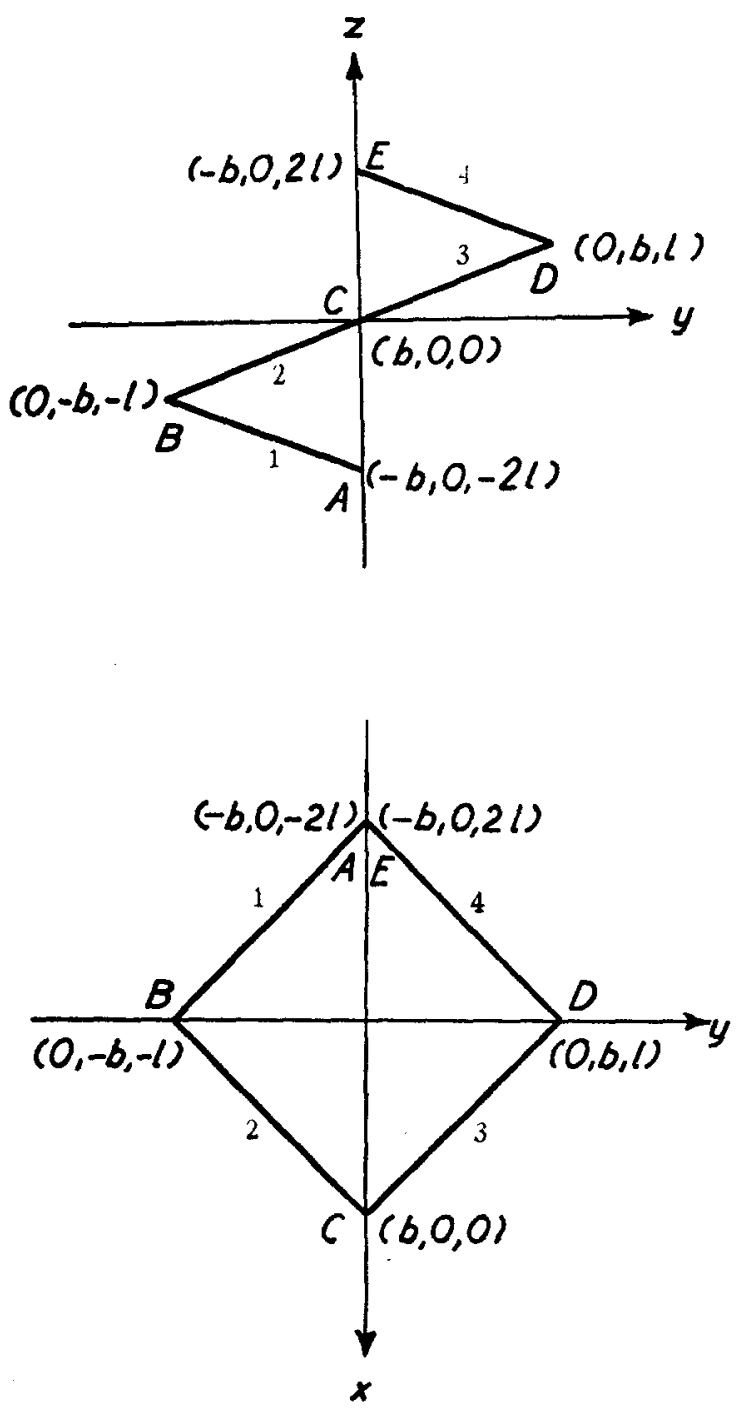

Fig. 2. Perpendicular projections of square, helical antenna with one turn. 
It follows from (4) that the electric field strength $\bar{E}$ may be obtained from the vector $\tilde{F}$, by multiplying $\bar{F}$ with a complex scalar $T$ depending on $r$, but being independent of $\theta$ and $\varphi$. For this reason we shall call $\vec{F}$ the normalized, electric field strength and $T$ the normalization constant. The problem of finding the far zone field from a given current distribution will be completely solved when we have found the normalization constant $T$ and the normalized, electric field strength $\bar{F}$.

In the present case of a single turn of a square helical antenna with the dimensions shown in Figs. 1 and 2 we find it convenient to choose

$$
K=\mu I_{0} b / 4 \pi \text {. }
$$

The normalization constant then becomes

$$
T=\frac{i k b \zeta I_{0} e^{i k r}}{4 \pi r} \text {. }
$$

Further, numbering the four straight pieces of wire, of which the antenna is composed, from 1 to 4 as shown in Fig. 2, we express the normalized, electric field strength as a sum of four parts

$$
\bar{F}=\sum_{\mu=1}^{4} \bar{F}^{\mu}
$$

where the $\mu$ th part arises from the current in the $\mu$ th wire of the antenna. We proceed to calculate the contribution to the normalized field strength coming from the first wire.

The straight piece of wire, 1 , may be expressed by the following parametric equations

$$
\left.\begin{array}{l}
x=-\frac{b}{2}+b t \\
y=-\frac{b}{2}-b t \\
z=-\frac{3 l}{2}+l t
\end{array}\right\}-\frac{1}{2} \leqq t \leqq \frac{1}{2}
$$

A line element $d \bar{s}$ of the antenna is then expressed by

$$
d \vec{s}=\left[\bar{i} \frac{d x}{d t}+\bar{j} \frac{d y}{d t}+\bar{k}-\frac{d z}{d t}\right] d t=[\bar{i} b-j b+\bar{k} l] d t,
$$

where $i, j$, and $\bar{k}$ are unit vectors pointing in the $x, y$, and $z$ directions. The arc length $s$ introduced above is expressed by the parameter $t$ in the following way,

$$
s=-\frac{3}{2} c+c t \text {. }
$$

For the current in conductor 1 we therefore have the following expression:

$$
I=I_{0} \exp \left[\underset{p}{i-\left(-\frac{3}{2} c+c t\right)}\right]
$$

The components of $\bar{F}^{1}$ may now be calculated by using the general formulas for $F_{x}$, etc., previously given in (8). Introducing the value of $K$ given in (9) we find

$$
\begin{aligned}
F_{x}= & \int_{-\frac{1}{2}}^{\frac{1}{3}} \exp \left\{i k \left[\frac{1}{p}\left(-\frac{3}{2} c+c t\right)\right.\right. \\
& -\left(-\frac{b}{2}+b t\right) \sin \theta \cos \varphi \\
& \left.\left.-\left(-\frac{b}{2}-b t\right) \sin \theta \sin \varphi-\left(-\frac{3}{2} l+l t\right) \cos \theta\right]\right\} d t,(16 \mathrm{a}) \\
F_{y}{ }^{1}= & -F_{x}^{1}, \\
F_{z}^{1}= & S F_{x}^{1} / 4 b
\end{aligned}
$$

In analogy with what has been done in the case of a circular helix ${ }^{4}$ we define the following secondary parameters:

$$
\begin{aligned}
& z=k b \sin \theta \\
& H=\frac{k}{2 \pi}\left[\frac{L}{p}-S \cos \theta\right]=\frac{1}{\lambda}\left[\frac{L}{p}-S \cos \theta\right] .
\end{aligned}
$$

By using these parameters we may express the above integral in the following way:

$$
\begin{aligned}
F_{x}{ }^{1}=\exp \left\{i-\left[-H \frac{3 \pi}{2}+z(\cos \varphi+\sin \varphi)\right]\right\} & \\
& \frac{\sin _{2}^{1}\left[H^{\pi}+z(-\cos \varphi+\sin \varphi)\right]}{\frac{1}{2}\left[H \frac{\pi}{2}+z(-\cos \varphi+\sin \varphi)\right]}
\end{aligned}
$$

The components of the normalized, electric field strength of the field emitted by each of the remaining three conductors may be calculated by using similar procedures. For conductor 2 we obtain $F_{x}{ }^{2}=F_{y}{ }^{2}$ $=4 b F_{z}^{2} / S$, where

$$
F_{x}^{2}=\exp \left\{\frac{1}{i-}\left[\begin{array}{c}
-H_{2}^{\pi}+z(-\cos \varphi+\sin \varphi) \\
2
\end{array}\right\}\right.
$$

$$
\times \frac{\sin \frac{1}{2}\left[H_{2}^{\pi}+z(-\cos \varphi-\sin \varphi)\right]}{\frac{1}{2}\left[\frac{\pi}{2}+z(-\cos \varphi-\sin \varphi)\right]}
$$


For conductor 3 we find $F_{x}{ }^{3}=-F_{y}{ }^{3}=-4 b F_{z}{ }^{3} / S$, where

$$
\begin{aligned}
& F_{x}^{3}=\exp \left\{i \frac{1}{2}\left[\begin{array}{c}
\pi \\
2
\end{array} \frac{\pi}{2}+z(-\cos \varphi-\sin \varphi)\right]\right\} \\
& \times \frac{\sin \frac{1}{2}[H-\pi+z(\cos \varphi-\sin \varphi)]}{\frac{1}{2}\left[\frac{\pi}{2}+z(\cos \varphi-\sin \varphi)\right]} .
\end{aligned}
$$

For conductor 4 is obtained $F_{x}^{4}=F_{y}^{4}=-4 b F_{z}^{4} / S$, where

$$
\begin{aligned}
& F_{x}{ }^{4}=\exp \left\{i \frac{1}{2}\left[H \frac{3 \pi}{2}+z(\cos \varphi-\sin \varphi)\right]\right\} \\
& \times \frac{\sin \frac{1}{2}\left[H-\frac{\pi}{2}+z(\cos \varphi+\sin \varphi)\right]}{\frac{1}{2}\left[H-\frac{\pi}{2}+z(\cos \varphi+\sin \varphi)\right]} .
\end{aligned}
$$

Expressions for the vectors $\bar{F}^{\mu}$ having been obtained, the normalized, electric field strength $\bar{F}$ is calculated from (11). It is convenient to introduce the parameters $v_{1}, \cdots, v_{6}$ through the equations

$$
\begin{aligned}
& v_{1}=\frac{1}{2}\left[H \frac{\pi}{2}+z(\cos \varphi+\sin \varphi)\right], \\
& v_{2}=\frac{1}{2}\left[H \frac{\pi}{2}+z(-\cos \varphi+\sin \varphi)\right], \\
& v_{3}=\frac{1}{2}\left[H \frac{\pi}{2}+z(\cos \varphi-\sin \varphi)\right], \\
& v_{4}=\frac{1}{2}\left[H \frac{\pi}{2}+z(-\cos \varphi-\sin \varphi)\right], \\
& v_{5}=\frac{1}{2}\left[H \frac{3 \pi}{2}+z(-\cos \varphi-\sin \varphi)\right], \\
& v_{6}=\frac{1}{2}\left[H \frac{3 \pi}{2}+z(\cos \varphi-\sin \varphi)\right] .
\end{aligned}
$$

By using these parameters we get

$$
\begin{aligned}
& F_{x}(\theta, \varphi)=\cos v_{5} \frac{\sin v_{2}}{v_{2}}+\cos v_{3} \frac{\sin v_{4}}{v_{4}}-\cos v_{4} \frac{\sin v_{3}}{v_{3}} \\
& -\cos v_{6} \frac{\sin v_{1}}{v_{1}}+i\left[-\sin v_{5} \frac{\sin v_{2}}{v_{2}}-\sin v_{3} \frac{\sin v_{4}}{v_{4}}\right. \\
& \left.-\sin v_{4} \frac{\sin v_{3}}{v_{3}}-\sin v_{3} \frac{\sin v_{1}}{v_{1}}\right] \text {, }
\end{aligned}
$$

$$
\begin{array}{r}
F_{y}(\theta, \varphi)=-\cos v_{5} \frac{\sin v_{2}}{v_{2}}+\cos v_{3} \frac{\sin v_{4}}{v_{4}}+\cos v_{4} \frac{\sin v_{3}}{v_{3}} \\
-\cos v_{6} \frac{\sin v_{1}}{v_{1}}+i\left[\sin v_{5} \frac{\sin v_{2}}{v_{2}}-\sin v_{3} \frac{\sin v_{4}}{v_{4}}\right. \\
\left.+\sin v_{4} \frac{\sin v_{3}}{v_{3}}-\sin v_{6} \frac{\sin v_{1}}{v_{1}}\right]
\end{array}
$$

$F_{z}(\theta, \varphi)=\frac{S}{4 b}\left\{\cos v_{5} \frac{\sin v_{2}}{v_{2}}+\cos v_{3} \frac{\sin v_{4}}{v_{4}}+\cos v_{4} \frac{\sin v_{3}}{v_{3}}\right.$

$$
\begin{gathered}
+\cos v_{8} \frac{\sin v_{1}}{v_{1}}+i\left[-\sin v_{5} \frac{\sin v_{2}}{v_{2}}-\sin v_{3} \frac{\sin v_{4}}{v_{4}}\right. \\
\left.\left.+\sin v_{4} \frac{\sin v_{3}}{v_{3}}+\sin v_{6} \frac{\sin v_{1}}{v_{1}}\right]\right\} .
\end{gathered}
$$

The components in the spherical coordinate system of the normalized, electric field strength $\bar{F}$ is obtained by substituting into (7) the above expressions for its components in the corresponding rectangular coordinate system.

\section{SQUARE HELICAL ANTENNA WITH $H=1$}

In the case of a single turn of a circular helical antenna with a uniformly progressing current wave the radiated field will be circularly polarized in the positive direction of the axis if the secondary parameter $H$,

$$
H=1 / \lambda[(L / p)-S \cos \theta],
$$

where the symbols $\lambda, p, L$, and $S$ have a meaning corresponding to the one attached to them in the case of the square helical antenna, is equal to 1 for $\theta=0 .^{3}$ In the present section we shall investigate the polarization in the positive direction of the axis of the field radiated from a single turn of a square helical antenna with a progressing current wave and with $H=1$ for $\theta=0$. For $H=1$ and $\theta=0$ we have $z=0$, from which it follows that $v_{1}=v_{2}=v_{3}=v_{4}=\pi / 2$ and $v_{5}=v_{6}=3 \pi / 4$. Inserting these values in (29) we hereby find for a square helix with a single turn,

$$
\begin{aligned}
& F_{x}(0, \varphi)=-i 8 / \pi, \\
& F_{y}(0, \varphi)=8 / \pi, \\
& F_{z}(0, \varphi)=0 .
\end{aligned}
$$

Substituting in the expression (7b) and (7c) for the $\theta$ and $\varphi$-components of the normalized electric field strength $\bar{F}$ we find

$$
\begin{aligned}
& F_{\theta}(0, \varphi)=\frac{8}{-i-e^{i \varphi}} \\
& \pi \\
& F_{\varphi}(0, \varphi)=-\frac{8}{\pi}
\end{aligned}
$$


The field in the positive direction of the axis for a square helix with a single turn and with $H=1$ for $\theta=0$ is thus seen to be circularly polarized. As in the case of a circular helix we conclude here that the field from a square helix with $H=1$ for $\theta=0$ and with an integral, but otherwise arbitrary number of turns, is circularly polarized in the direction $\theta=0$ and that the field in this direction from a square helix with $H=1$ for $\theta=0$ and with a large but not necessarily integral number of turns is approximately circularly polarized. ${ }^{3,4}$

\section{SPECIAL CASES OF A SQUARE HELICAL ANTENNA}

The square helical antenna treated above involves as special cases a rectilinear wire with a progressing current wave and a small square loop carrying a constant current. As a controlling measure and in order to demonstrate the use of the formulas for a square helical antenna of arbitrary dimensions as developed above, these formulas will be applied to the two special cases mentioned here.

\section{Rectilinear Antenna}

A rectilinear antenna with the length $L$ placed so as to coincide with the part of the $z$ axis between $z=-(L / 2)$ and $z=(L / 2)$ and carrying a current wave progressing in the positive $z$ direction, $I=I_{0} \exp [i(k z / p)]$, may be obtained from a square helical antenna with one turn, with the length $L$ and with $b=0$, where $2 b$ denotes the diameter of the square cross section of the cylinder on which the helix is situated. With the notation introduced above we have for such a helical antenna that $b=0$, $S=L$, and $z=0$, from which it follows that $v_{1}=v_{2}=v_{3}$ $=v_{4}=v=H(\pi / 4)$ and $v_{5}=v_{6}=3 v$. The components $F_{x}$ and $F_{y}$ give no contributions to the field components, as they are finite, and as they are to be multiplied by the normalization constant $T$ containing $b^{1}$, which is zero. The component $F_{z}$ contains $b^{-1}$, and therefore, when multiplied by $T$, gives a finite contribution to the field components. We find

$$
F_{z}=\frac{L}{4 b} \frac{2 \sin v}{v}(\cos 3 v+\cos v)=\frac{S \sin 4 v}{b} \frac{1 v}{4 v} .
$$

Substituting this expression in (7) we get

$$
\begin{aligned}
& F_{\theta}=-L \sin 4 v \sin \theta / b 4 v, \\
& F_{\varphi}=0,
\end{aligned}
$$

from which we obtain by using (4)

$$
\begin{aligned}
& E_{r}=E_{\varphi}=0, \\
& E_{\theta}=-\frac{\left.i \zeta I_{0} e^{i k r} \sin (k S / 2)[(1 / p)-\cos \theta)\right]}{2 \pi r} .
\end{aligned}
$$

This is just the well-known expression for the field in the far zone from a vertical antenna with a progressing current wave. $^{7}$

\section{Small Square Loop with Constant Current}

A square loop with the diameter $2 b \ll \lambda$ placed in the $(x, y)$ plane with its center at origo and carrying a constant current $I_{0}$ may be obtained from a square helical antenna with one turn, with the height $S$ equal to zero, and with the transmission coefficient $\beta$ equal to zero. For such a helical antenna we have, with the notation introduced above, that $S=0, z=k b \sin \theta \ll 1$, and $H=0$, from which it follows that $v_{1}=-v_{4}=-v_{5}=(z / 2)$ $X(\cos \varphi+\sin \varphi) \ll 1$ and $v_{2}=-v_{3}=-v_{6}=(z / 2)(-\cos \varphi$ $+\sin \varphi) \ll 1$. From this we find the following expressions for the rectangular components of the normalized, electric field strength,

$F_{x}=i 2 \sin v_{1} \sin v_{2}\left[\frac{1}{v_{1}}+\frac{1}{v_{2}}\right]$

$$
\cong i 2\left[v_{1}+v_{2}\right]=i z 2 \sin \varphi
$$

$F_{y}=i 2 \sin v_{1} \sin v_{2}\left[\frac{1}{v_{1}}-\frac{1}{v_{2}}\right]$

$$
\cong i 2\left[-v_{1}+v_{2}\right]=-i z 2 \cos \varphi
$$

$F_{z}=0$

After substituting in Eq. (7) we find $F_{\theta}=0$ and

$$
F_{\varphi}=-i 2 z=-i 2 k b \sin \theta \text {. }
$$

Using (4) we finally obtain $E_{r}=E_{\theta}=0$ and

$$
E_{\varphi}=\frac{k^{2} A \zeta I_{0} e^{i k r}}{4 \pi r} \sin \theta
$$

where $A=2 b^{2}$ is the area of the square loop. This is the well-known expression for the far zone field radiated by a constant current in a plane loop with the area $A$ and with a diameter that is small compared to the wavelength. ${ }^{8}$

\section{SQUARE, NUMERICAL EXAMPLE OF A HELICAL ANTENNA}

The application of the above derived formulas will be demonstrated by a numerical example. The field will be calculated for a single turn of a helix with the same data as one of the helices used by Kraus., ${ }^{2,3}$ Using the above introduced notation we may describe this helix by the following data, denoting by $N$ the number of turns: $S=15.025 \mathrm{~cm}, L=72.26 \mathrm{~cm}, \lambda=66.67 \mathrm{~cm}$, and $N=7$. From these values of $S$ and $L$ we find $b=12.456 \mathrm{~cm}$ and $l=3.756 \mathrm{~cm}$. For the parameter $p$, defined as the

${ }^{7}$ J. D. Kraus, Antennas (McGraw-Hill Book Company, Inc., New York, 1950), pp. 148-153.

${ }^{8}$ See reference 7 , pp. 155-157. 
quotient between the specific transmission coefficient of the medium surrounding the antenna and the transmission coefficient of the current wave, $\mathrm{Kraus}^{3}$ gives the following semi-empirical formula occurring in Hansen and Woodyard's ${ }^{9}$ theory of a homogeneous, linear array

$$
p=\frac{L / \lambda}{(S / \lambda)+1+(1 / 2 N)}
$$

For a helical antenna with the above given data we find $p=0.8358$. From the primary constants of the helical antenna in question we calculate the following secondary constants $z=67^{\circ} .259 \sin \theta$ and $H=1.2968$ $-0.2254 \cos \theta$.

By using the above given parameters we first calculate the $x, y$, and $z$ components of the normalized, electric field strength $\bar{F}$ and then the $\theta$ - and $\varphi$-components of $\bar{F}$. The normalization constant being given by (10), the electric field strength $\bar{E}$ is then completely determined. In the following section we shall compare the field radiated from the square helical antenna considered in this section with the field radiated from an equivalent circular helical antenna having the same length and the same pitch and also the same current distribution along the wire as the square helix in question. In order to be able to compare the fields radiated from the two antennas we shall use the same normalization constant in both cases, viz.,

$$
T=i k a \zeta I_{0} e^{i k r} / 4 \pi r
$$

where $a$ denotes the radius of the cylinder on which the circular helix is situated. The normalized, electric field strength $\bar{F}^{*}$ corresponding to the normalization constant $(40)$ is determined by $\bar{F}^{*}=b \bar{F} / a$. Through a simple calculation we find $b / a=1.1107$.

Calculations of $F_{\theta}{ }^{*}$ and $F_{\varphi}{ }^{*}$ are made for $\varphi=0^{\circ}, 90^{\circ}$, $180^{\circ}$, and $270^{\circ}$. For each of these cases the real and imaginary parts of $F_{\theta}{ }^{*}$ and $F_{\varphi}{ }^{*}$ are calculated and plotted for $0^{\circ}<\theta<180^{\circ}$. In Fig. $3 \dagger$ are plotted the real and the imaginary parts of $F_{\theta}^{*}$ as functions of $\theta$ for $\varphi=0^{\circ}$ and $\varphi=180^{\circ}$. In Fig. 4 are plotted the same functions for $\varphi=90^{\circ}$ and $\varphi=270^{\circ}$; for these values of $\varphi$ the imaginary part of $F_{\theta}{ }^{*}$ is zero. The real and the imaginary parts of $F_{\varphi}{ }^{*}$ are plotted in Fig. 5 as functions of $\theta$ for $\varphi=0^{\circ}$ and $\varphi=180^{\circ}$. In Fig. 6 are plotted the same functions for $\varphi=90^{\circ}$ and $\varphi=270^{\circ}$; for these values of $\varphi$ the real part of $F_{\varphi}{ }^{*}$ is zero.

Kraus has used a simplified theory to calculate the field from a single turn of a helical antenna ${ }^{3}$ which hasas far as the author can find out-the same data as the helix in the present numerical example, except that Kraus' antenna is a left-handed screw, whereas the

${ }^{9}$ W. W. Hansen and J. R. Woodyard, Proc. Inst. Radio Engrs. 26, 333-345 (1938).

† In Fig. 15 of the author's 1950 paper (reference 4), which corresponds to Fig. 3 of this paper, "Re" and " $I m$ " should be interchanged. This error was kindly pointed out to the author by Professor J. D. Kraus. present one is right-handed. In the notation of the present paper, Kraus has calculated and plotted the field components $E_{\theta}$ and $E_{\varphi} \ddagger$ for the azimuthal angles $\varphi=90^{\circ}$ and $\varphi=270^{\circ}$. The reflected image in the axis $\theta=0$ of the curves obtained by Kraus are shown in Figs. 7 and 8. The reflection takes care of the opposite winding directions of the two helices. Except for the scale factor the curves in Figs. 4 and 7 showing the $\theta$-component of the field should be identical, and so should the curves in Figs. 6 and 8 showing the $\varphi$-component. As may be seen from the figures there is not only a qualitative, but even a fair quantitative agreement between Kraus' approximate expression for the field from a square helix with a uniformly progressing current wave and the exact expression for the field given in this paper. No great error seems therefore to be committed in applying Kraus' approximate method of calculation for a rough estimation of the field from a single turn of a square helix. However, besides being more exact the present method has the advantage of greater clearness and simplicity and should therefore be preferred even for rough calculations. As Kraus ${ }^{3}$ points out, for a helical antenna with several turns the effect upon the total field of the field from a single turn is overshadowed by the influence of the array characteristic. However, the polarization of the field from a helical antenna is exactly the same as the polarization of the field from a single turn of the antenna. In investigations of helical antennas it is therefore important that reliable formulas for the field from a single turn are available.

Kraus' approximate method $^{2}$ of calculating the field from a circular helical antenna involves two steps: (1) the circular helix is replaced by an equivalent square helix, (2) various mathematical approximations are introduced in the calculation of the field from the square, helical antenna. The legitimacy of the last step was discussed above. In the next section we shall investigate whether the first step is justified or not, by plotting curves of the field components of a circular, helical antenna equivalent to the square, helical antenna considered in the above numerical example, and comparing the curves calculated in this way with the curves obtained for the square helix.

\section{THE FIELD RADIATED BY AN EQUIVALENT CIRCULAR HELICAL ANTENNA}

Using the definition of equivalence of a square and a circular helical antenna given by Kraus ${ }^{2}$ we investigate in this section the field from a circular helical wire with the same length, the same pitch, and the same current distribution as the square helix in the example given above. For this purpose we use the exact formulas for

$\ddagger$ In Fig. 9 of Kraus' 1949 paper (reference 3) it seems that the symbols $E_{\theta T}$ and $E_{\phi T}$ should be interchanged. The mistake probably has its origin in the circumstance that the curves in the aforementioned paper were calculated on the basis of the method of calculation given in Kraus' 1948 paper (reference 2), where the helix had an orientation different from the one occurring in the first-mentioned paper. 


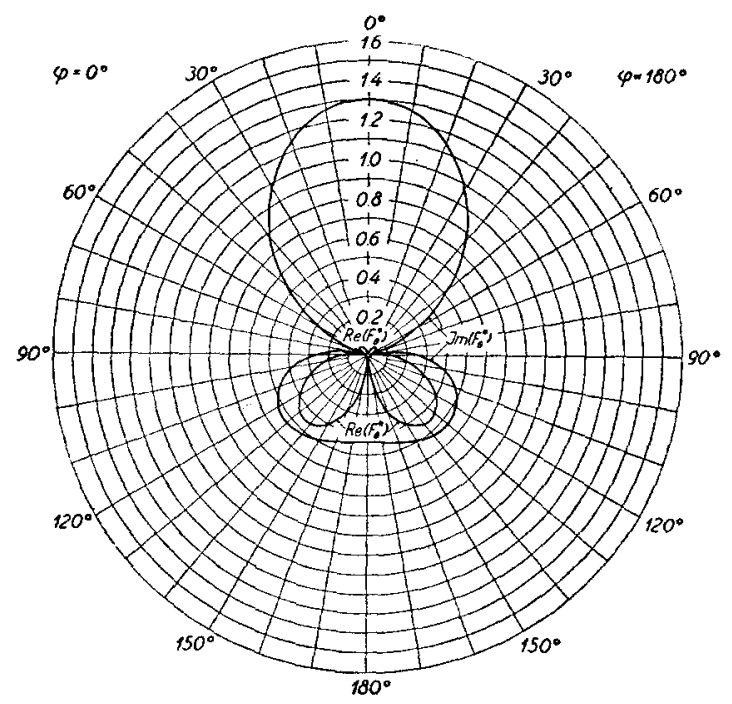

F1G. 3. The real and the imaginary part of the $\theta$-component, $F_{\theta}{ }^{*}$, of the normalized, electric field strength of the field from the square helical antenna, plotted as functions of $\theta$ for $\varphi=0^{\circ}$ and $180^{\circ}$. The curves were calculated according to the rigorous formulas of this paper.

the field radiated by a circular helix with a progressing current wave, developed by the author ${ }^{4}$ and independently by Kornhauser. ${ }^{5}$ With the normalization constant $T$ given by (40) calculations of the $\theta$ - and $\varphi$-components of the normalized, electric field strength $\bar{F}$ are made for $\varphi=0^{\circ}, 90^{\circ}, 180^{\circ}$, and $270^{\circ}$. For each of these cases the real and imaginary parts of $F_{\theta}$ and $F_{\varphi}$ are calculated and plotted for $0^{\circ}<\theta<180^{\circ}$ in Figs. 9-12. There is a one to one correspondence between these four figures and the Figs. 3-6 in the mentioned order. A comparison between the plotted field components for

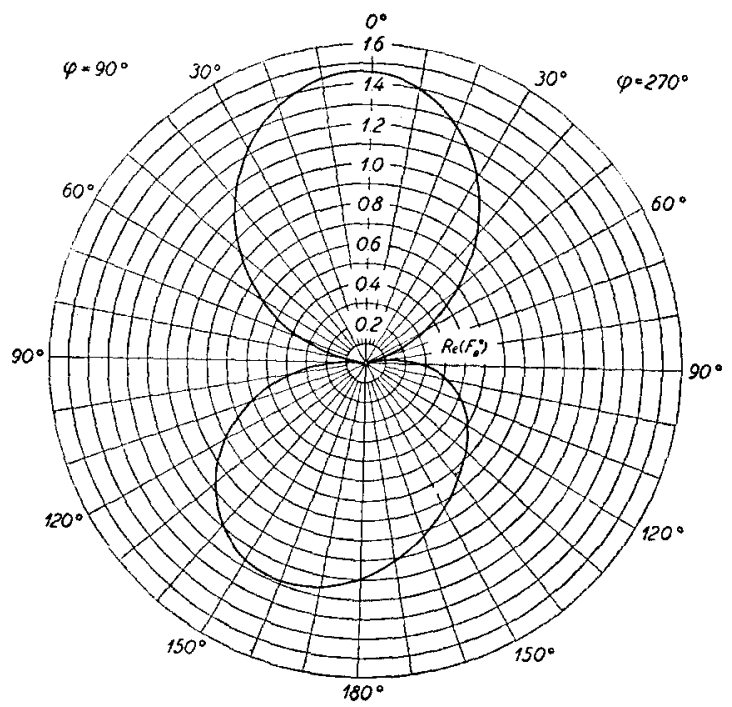

FIG. 4. The real part of the $\theta$-component $F_{\theta}{ }^{*}$ of the normalized, electric field strength of the field from the square helical antenna, plotted as a function of $\theta$ for $\varphi=90^{\circ}$ and $270^{\circ}$; the imaginary part of $F_{\theta}{ }^{*}$ is zero for these values of $\varphi$. The curve was calculated according to the rigorous formulas of this paper.

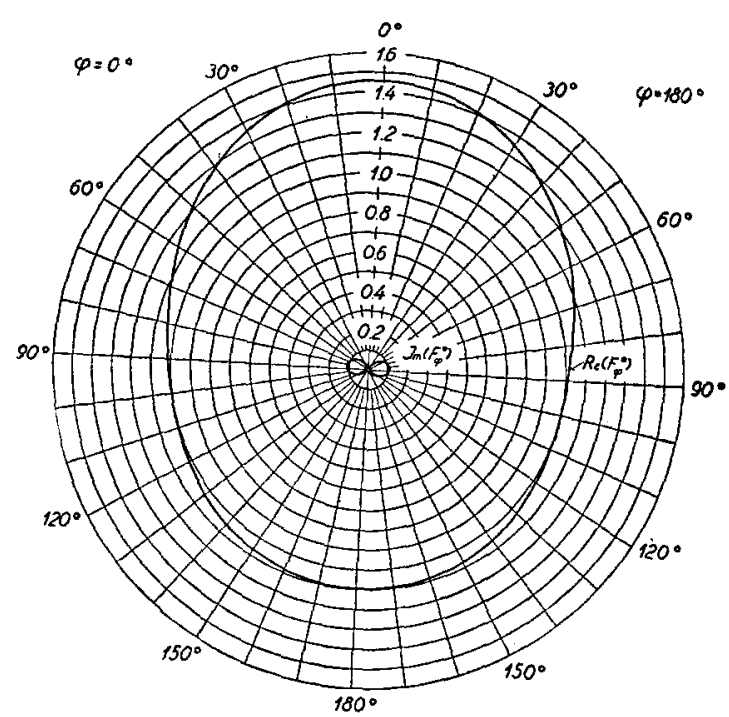

FIG. 5. The real and the imaginary part of the $\varphi$-component $F_{\varphi}{ }^{*}$ of the normalized, electric field strength of the field from the square helical antenna, plotted as functions of $\theta$ for $\varphi=0^{\circ}$ and $180^{\circ}$. The curves were calculated according to the rigorous formulas of this paper.

the square and for the circular helical antenna show that there is a good qualitative and a fair quantitative agreement between the fields from the two equivalent antennas. It seems therefore that also the first step in Kraus' approximate calculation of the field radiated by a circular helical antenna is justified. However, as was pointed out by the author ${ }^{4}$ and also by Kornhauser, ${ }^{5}$ the exact formulas are clearer and simpler than Kraus' approximate formulas.

By considering the Figs. 9-12 we are now able to state the reason why Kornhauser ${ }^{5}$ finds a considerable

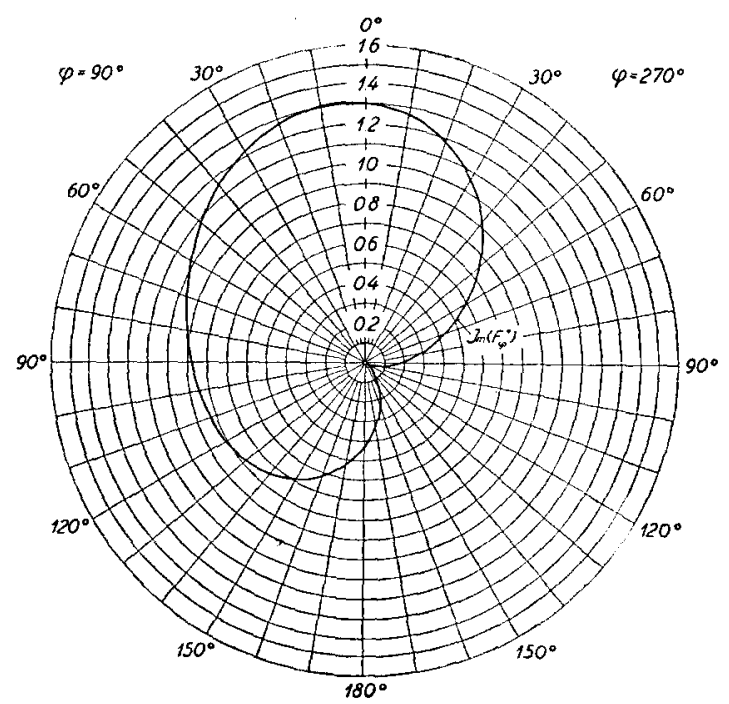

Fig. 6. The imaginary part of the $\varphi$-component, $F_{\varphi}{ }^{*}$ of the normalized, electric field strength of the field from the square helical antenna, plotted as a function of $\theta$ for $\varphi=90^{\circ}$ and $270^{\circ}$; the real part of $F_{\varphi}^{*}$ is zero for these values of $\varphi$. The curve was calculated according to the rigorous formulas of this paper. 


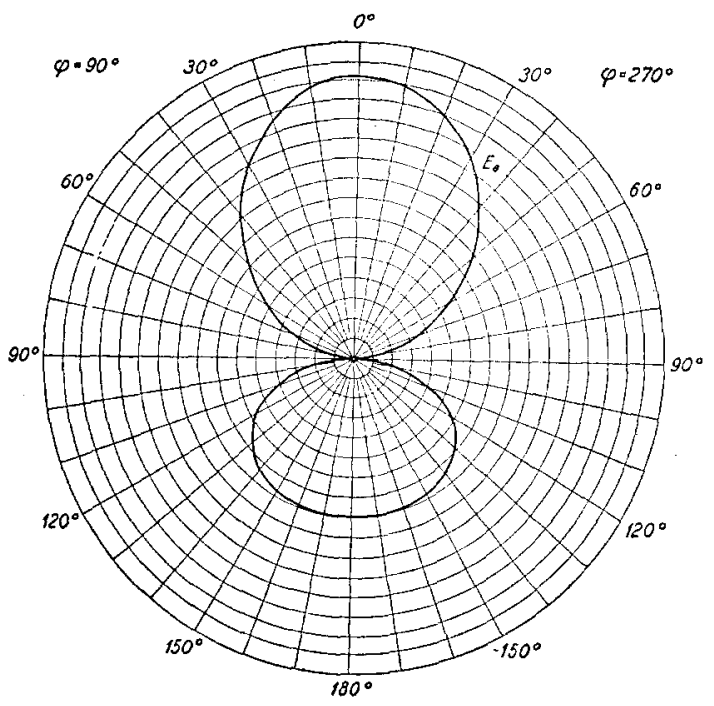

Fig. 7. The $\theta$-component $E_{\theta}$ of the electric field strength of the field from the circular helical antenna, plotted as a function of $\theta$ for $\varphi=90^{\circ}$ and $270^{\circ}$. The curve was calculated according to Kraus' approximate formulas.

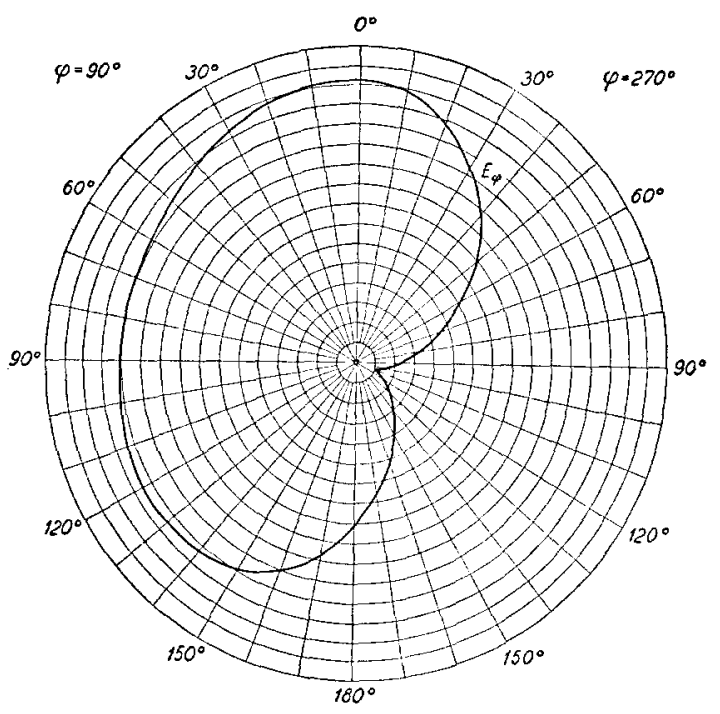

FIG. 8. The $\varphi$-component $E_{\varphi}$ of the electric field strength of the field from the circular helical antenna plotted as a function of $\varphi$ for $\varphi=90^{\circ}$ and $270^{\circ}$. The curve was calculated according to Kraus' approximate formulas.

disagreement between the curves showing the field components calculated by using the exact formulas and the curves showing the field components calculated by using Kraus' approximate method. In the notation used in this paper Kornhauser compares in Fig: 4 of his paper the field components in the plane $\varphi=0^{\circ}$ and $\varphi=180^{\circ}$ calculated by the exact method with the field components in the plane $\varphi=90^{\circ}$ and $\varphi=270^{\circ}$ calculated by Kraus' method. The two sets of curves do not agree, and they should not.

\section{CONCLUSION}

For mechanical reasons a helical beam antenna for use in the meter-band is constructed as a square helix of wire. As shown by Kraus the current distribution on a helical antenna may approximately be described as a uniformly progressing current wave with a constant amplitude. In the present paper rigorous formulas are derived for the components of the field radiated by a square helical antenna with a current distribution of

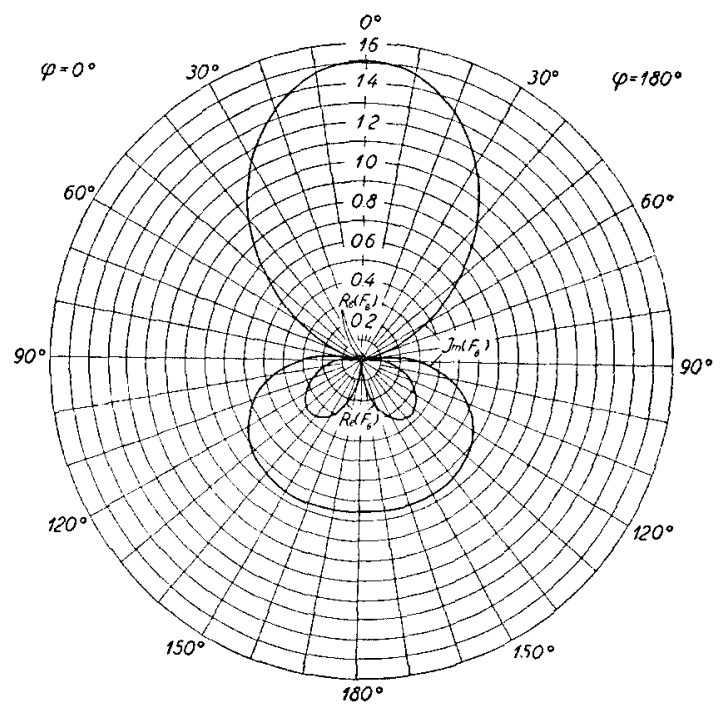

FIG. 9. The real and the imaginary part of the $\theta$-component $F_{\theta}$ of the normalized, electric field strength of the field from the circular helical antenna, plotted as functions of $\theta$ for $\varphi=0^{\circ}$ and $180^{\circ}$. The curves were calculated according to the rigorous formulas derived in a previous paper by the author.

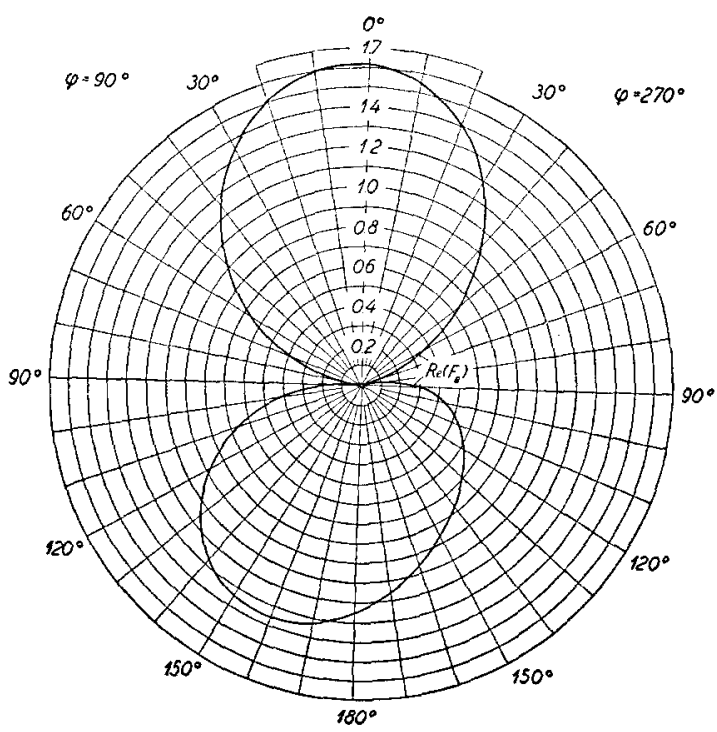

FIG. 10. The real part of the $\theta$-component $F_{\theta}$ of the normalized, electric field strength of the field from the circular helical antenna, plotted as a function of $\theta$ for $\varphi=90^{\circ}$ and $270^{\circ}$; the imaginary part of $F_{\theta}$ is zero for these values of $\varphi$. The curve was calculated according to the rigorous formulas derived in a previous paper by the author. 


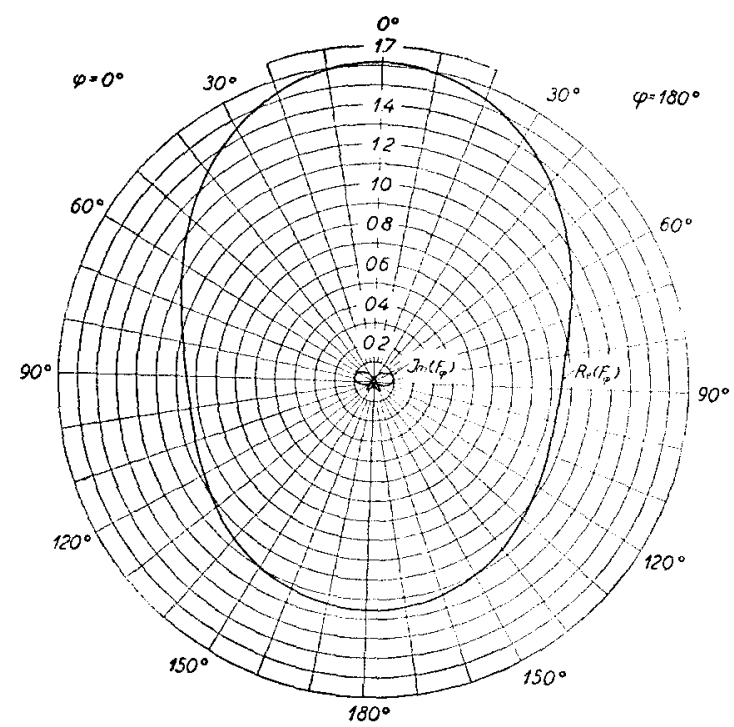

FIG. 11. The real and imaginary parts of the $\varphi$-component $F_{\varphi}$ of the normalized, electric field strength of the field from the circular helical antenna, plotted as functions of $\theta$ for $\varphi=0^{\circ}$ and $180^{\circ}$. The curves were calculated according to the rigorous formulas derived in a previous paper by the author.

this type. These rigorous formulas turn out to be simpler than the approximate formulas derived by Kraus. Used on a numerical example the two sets of formulas show good agreement with each other.

In Kraus' approximate theory of the field from a circular helical antenna he first replaces the circular helix with an equivalent square helix having the same length, pitch, and current distribution. Using formulas derived in a previous paper by the author and independently by Kornhauser, the field radiated by a circular helical antenna equivalent to the square, helical antenna occurring in the above-mentioned, numerical example, was calculated. There is a good agreement, qualitatively as well as quantitatively, between the curves showing the components of the field from the equivalent, circular helix and from the original, square helical antenna. This points to the legitimacy of the first step in Kraus' approximate method of calculation.

By using the exact formulas for the field radiated by a

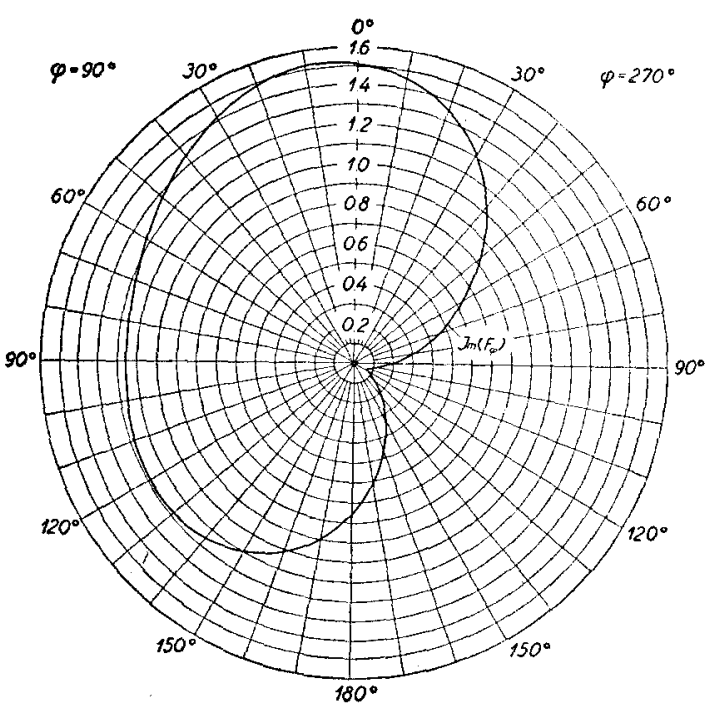

Fig. 12. The imaginary part of the $\varphi$-component $F_{\varphi}$ of the normalized, electric field strength of the field from the circular helical antenna, plotted as a function of $\theta$ for $\varphi=90^{\circ}$ and $270^{\circ}$; the real part of $F_{\varphi}$ is zero for these values of $\varphi$. The curve was calculated according to the rigorous formulas derived in a previous paper by the author.

circular helical antenna, Kornhauser comes to a result in apparent conflict with the corresponding result obtained by Kraus' approximate method. It is shown in the present paper that probably this disagreement is because of the fact that Kornhauser compares the field components in one plane, calculated according to the rigorous method, with the field components in a plane perpendicular to the first plane, calculated by using Kraus' approximate method.

\section{ACKNOWLEDGMEN'T}

The investigation presented here was carried out at the Microwave Laboratory of the Royal Technical University of Denmark, with a grant from the Research Council of the Technical Sciences in Denmark. I am indebted to the head of the Microwave Laboratory, Professor J. Oskar Nielsen for permission to publish this paper. 